\title{
Nutzen und Aufwand von Qualitätsaktivitäten: Ein neues Tool bringt Licht ins Dunkel
}

\author{
Qualitätsaktivitäten sind aus dem Alltag von Ärztinnen und Ärzten nicht wegzuden- \\ ken. Was diese Aktivitäten aber tatsächlich an Nutzen und an Aufwand bedeuten, \\ ist bis heute in den meisten Fällen nicht ermittelt. Die Schweizerische Akademie für \\ Qualität in der Medizin SAQM hat sich deshalb mit einem interdisziplinären Projekt- \\ team das Ziel gesetzt, ein praxisnahes und pragmatisches Bewertungsinstrument \\ aus der Praxis für die Praxis zu entwickeln.
}

Hélène Beutler ${ }^{b}$,

Johannes Brühwiler ${ }^{\text {, }}$

Christoph Gehrlach ${ }^{\text {, }}$

Martina Herspergera,

Rebekka Kurz ${ }^{\text {, }}$ Varja Meyera,

Adrian Rohrbassere

(Projektteam, alphabetisch)

a $\mathrm{FMH}$, Schweizerische Akademie für Qualität in der Medizin SAQM (Projektleitung)

b Verbindung der psychiatrischpsychotherapeutisch tätigen Ärztinnen und Ärzte der Schweiz FMPP

c Hausärzte Schweiz MFE

d Kompetenzzentrum Qualitätsmanagement, Berner Fachhochschule

e Schweizerische Gesellschaft für Allgemeinmedizin SGAM

Korrespondenz: FMH, SAQM, Abteilung DDQ Elfenstrasse 18

CH-3000 Bern 15 Tel. 0313591111 Fax 0313591112 saqm[at]fmh.ch

\section{Ausgangslage}

Die Resultate des Projekts «Q-Monitoring ambulante Medizin $\mathrm{CH}$ ", das die Abteilung Daten, Demographie und Qualität DDQ der FMH zusammen mit zehn medizinischen Fachgesellschaften durchführte, zeigen eindrucksvoll, dass die Bandbreite von Qualitätsaktivitäten (wie beispielsweise Patientenbefragungen, Fortbildungen, Qualitätszirkel usw.) gross ist [1]. Viele dieser Aktivitäten helfen, die Prozesse in der Praxis zu optimieren und Fehler zu vermeiden. Da solche Aktivitäten für Ärztinnen und Ärzte meistens aber auch einen zusätzlichen Aufwand bedeuten (z.B. zeitlicher oder finanzieller Art), ist es umso wichtiger, sich über deren Aufwand und Nutzen Gedanken zu machen und diese im jeweiligen fachlichen und praxisbezogenen Kontext genau zu analysieren.

Parallel zu den Arbeiten am Projekt Q-Monitoring hat die DDQ deshalb die Literatur zum Thema Aufwand und Nutzen von Qualitätsaktivitäten analysiert und darauf basierend das Grundlagenpapier «Was bringt die Qualitätsarbeit den Patienten und den Ärzten?» [2] publiziert. Zusammengefasst konnte gezeigt werden, dass die Thematik um Aufwand und Nutzen von Qualitätsaktivitäten nicht einfach ist, da sich vor allem viele der Aufwand- und Nutzenfaktoren nicht oder nur schwer in Geld messen lassen. Weiter sind Studien, die diese Faktoren in eine Relation zueinander setzen, zum heutigen Zeitpunkt rar. Trotz der Komplexität des Themas lassen sich bei Qualitätsaktivitäten jedoch die einzelnen Aufwand- und Nutzenaspekte ermitteln und gewissen Kategorien zuordnen. Da sich Aufwand- und Nutzenfaktoren aber wie gesagt nicht oder nur schwer finanziell quantifizieren lassen, muss die Beurteilung des Arztes berücksichtig werden, der die Qualitätsarbeit aufgrund seiner klinischen, praktischen und organisatorischen Erfahrung einschätzt.

\section{Projektstart}

Die gewonnenen Erkenntnisse veranlassten die DDQ, zusammen mit Vertretern der am Pilotprojekt Q-Monitoring beteiligten Fachgesellschaften und Experten des Kompetenzzentrums Qualitätsmanagement der Berner Fachhochschule eine Projektgruppe (vgl. Autorenschaft) zu bilden. Die Absicht war, sich mit der Bewertung von Aufwand und Nutzen von Qualitätsaktivitäten detaillierter zu befassen. Sie lancierte 2010 das Pilotprojekt «Bewertung und Förderung von Qualitätsaktivitäten BFQA» mit dem Ziel, ein pragmatisches, praxisnahes Bewertungsraster für die Beurteilung von Qualitätsaktivitäten nach deren Aufwand und Nutzen zu entwickeln und zu testen. In verschiedenen Fokusgruppen wurden die Einschätzungen von in der Praxis tätigen Ärzten miteinbezogen und das Raster nach und nach zu einem Bewertungsinstrument entwickelt. Der Prozess wurde dabei stets vom Bedürfnis geleitet, eine Methode zu schaffen, mit der die gezwungenermassen eher subjektiven Aufwand-Nutzen-Bewertungen zu möglichst objektiven und allgemeingültigen Aussagen umgewandelt werden können. Solche Aussagen könnten dann als Entscheidungsgrundlage für oder gegen die Durchführung einer Qualitätsaktivität verwendet werden. Im Verlauf des Projekts wurde der Projektname konkretisiert und in den heute gültigen Namen «Aufwand-Nutzen-Analyse von Qualitätsaktivitäten $\mathrm{AQA}$ » geändert.

\section{Aufwand-Nutzen-Analyse von Qualitäts- aktivitäten: Ziele und Herausforderungen}

Ziel der Aufwand-Nutzen-Analyse von Qualitätsaktivitäten AQA ist es, anhand eines praxisnahen und strukturierten Bewertungsprozesses (Abb. 1, nächste Seite) Qualitätsaktivitäten aus dem ambulanten medizinischen Alltag nach deren Aufwand und Nutzen zu analysieren. Dadurch soll das Aufwand-Nutzen- 


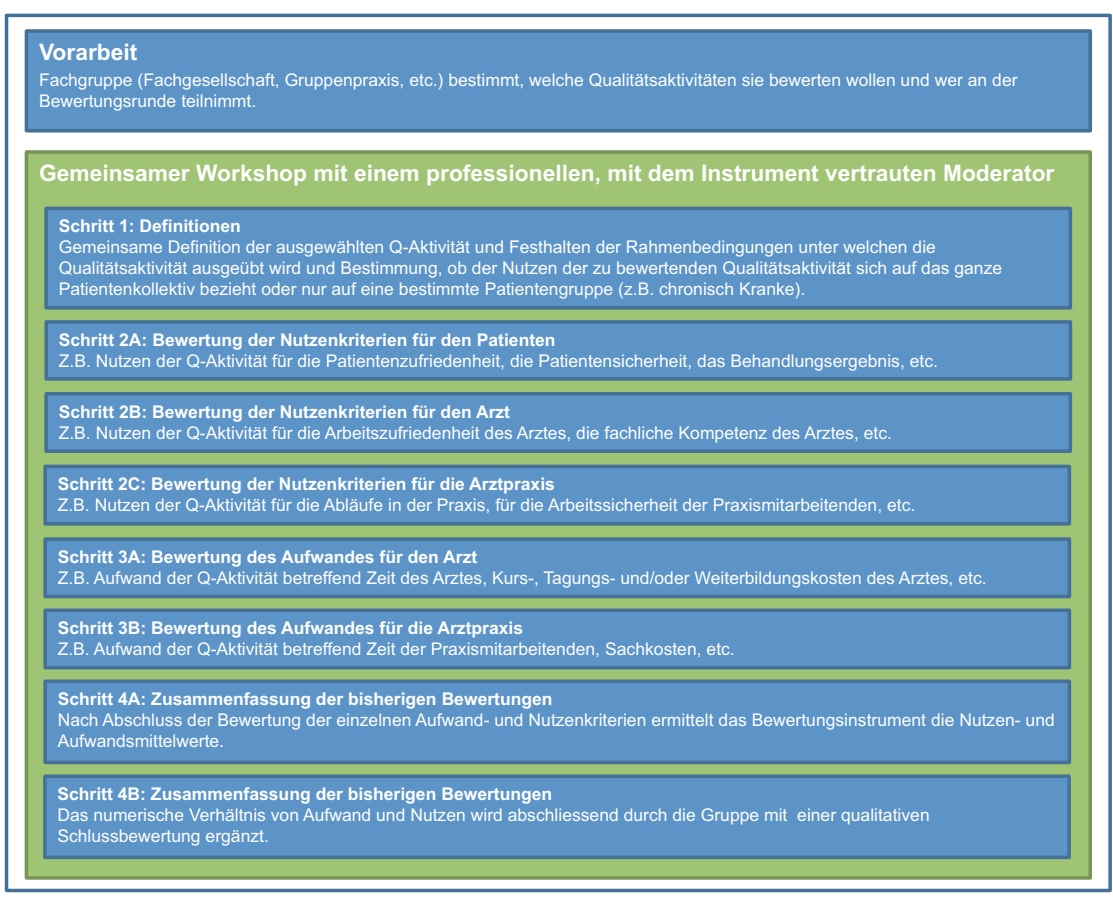

Abbildung 1

Ablaufschema der Aufwand-Nutzen-Analyse von Qualitätsaktivitäten.

Verhältnis einzelner Qualitätsaktivitäten sichtbar gemacht werden. Bei Bedarf können so beispielsweise Fachgesellschaften oder Gruppenpraxen entsprechende Qualitätsaktivitäten fördern.

Zahlreiche Diskussionen in der Projektgruppe wie auch in Fokusgruppen zeigten auf, dass die Entwicklung eines solchen Instruments eine Herausforderung darstellt. Problempunkte waren u. a.:

- die Verständlichkeit und Anwendbarkeit

- der geeignete Reduktionsgrad

- die seltene Einigkeit in der Bewertung etwa aufgrund der unterschiedlichen Vorerfahrungen und Arbeitsorte der Teilnehmenden

- der Umgang mit unterschiedlichem Begriffsverständnis und mit der Tatsache, dass die Einschätzungen am Schluss immer abhängig sind von der Tätigkeit und Erfahrung des Praktikers

Wiederholt wurde aber klar, dass die Anwendung eines solchen Instruments auch der Qualitätsentwicklung und insbesondere der strukturierten Meinungsbildung in einer Gruppe und somit der Orientierung dient und nicht nur der qualitativen und quantitativen Bewertung von Aktivitäten. Somit verfolgt das Instrument die beiden Ziele «Definieren von Nutzen und Aufwand einer ausgewählten Qualitätsaktivität» und «Förderung des Meinungsbildungsprozesses zu einer ausgewählten Qualitätsaktivität», wobei je nach Gruppe eines davon mehr im Vordergrund stehen kann. Der entwickelte Bewertungsprozess wurde während der Projektarbeiten immer wieder als wichtig, anregend sowie für die weiteren Diskussionen punkto Qualitätsfragen innerhalb der Ärzteschaft als sehr wertvoll eingestuft.

\section{Ablauf der Aufwand-Nutzen-Analyse}

Vor der eigentlichen Bewertung bestimmt eine interessierte Gruppe von Fachpersonen (z. B. eine Fachgesellschaft, eine Gruppenpraxis oder ein Qualitätszirkel), welche Qualitätsaktivität sie bewerten möchte. Der anschliessende Bewertungsprozess findet zusammen mit einem professionellen und mit dem Instrument vertrauten Moderator im Rahmen einer strukturierten Gruppendiskussion statt. Der genaue Ablauf ist in Abbildung 1 dargestellt. Idealerweise setzt sich die Gruppe aus vier bis acht Fachpersonen zusammen, die mit dem Thema Qualität im Allgemeinen und mit den zu bewertenden Qualitätsaktivitäten im Speziellen vertraut sind.

\section{Aktueller Projektstand}

Das Bewertungstool «Aufwand-Nutzen-Analyse von Qualitätsaktivitäten AQA» wird im Moment von verschiedenen Fachgruppen in der Praxis getestet. Nach einer Evaluation dieser Testanwendungen wird entschieden, ob das Instrument weiteren interessierten Fachgruppen zur Verfügung gestellt wird.

Weitere Informationen wie das Faktenblatt oder Informationen für interessierte Fachgruppen können auf www.saqm.ch (Themenfelder $\rightarrow$ Qualitätsprojekte $\rightarrow$ Aufwand-Nutzen-Analyse von Qualitätsaktivitäten) heruntergeladen werden.

\section{Literatur}

1 Meyer-Nikolic V, Hersperger M. Qualitätsentwicklung in der ambulanten Medizin CH. Q-MonitoringResultate schaffen Übersicht. Schweiz Ärztezeitung. 2012;93(27/28):1036-8.

2 Nikolic V, Herren D. Was bringt die Qualitätsarbeit den Patienten und den Ärzten? Schweiz Ärztezeitung. 2010;91(43):1679-83. 